\title{
Microwave Assisted One Pot Synthesis of Functionalized Pyrrole Derivatives Catalyzed by Uranyl Nitrate Hexa Hydrate
}

\author{
Dr. K. Venkatesan ${ }^{1}$, Dr. Pagadala Ramakanth ${ }^{2}$ and Dr. Ch. Anjaneyulu ${ }^{3}$ \\ ${ }^{1}$ Asst. Professor, CVR College of Engineering/H\&S Department (Chemistry), Hyderabad, India \\ Email: venkippk@gmail.com \\ ${ }^{2}$ Asst. Professor, CVR College of Engineering/H\&S Department (Chemistry), Hyderabad, India \\ Email: pagadalaramakanth@gmail.com \\ ${ }^{3}$ Professor, CVR College of Engineering/H\&S Department (Chemistry), Hyderabad, India \\ Email: anjaneyulu_22@yahoo.co.in
}

\begin{abstract}
An effective and simple method for the functionalized pyrrole synthesis has been developed using a $\mathrm{UO}_{2}\left(\mathrm{NO}_{3}\right)_{2} \cdot 6 \mathrm{H}_{2} \mathrm{O}$ catalyst under conventional method. Pyrrole synthesis with uranyl nitrate hexa hydrate catalyst has various advantages, such as fast activity, good yields and reduces reaction times in ethanol media. The synthesized target compounds were characterized by FT-IR, elemental analysis, ${ }^{1} \mathrm{H}$ and ${ }^{13} \mathrm{C}$ NMR.
\end{abstract}

Index Terms: Microwave, Uranyl nitrate, Pyrrole.

\section{INTRODUCTION}

The heterocyclic compounds contain nitrogen atom in their structures which provide a wide array of biological activities; the valence electron of nitrogen atom contribute to promoting the building of several supramolecular structures. Pyrrole moieties are among the most important compound in heterocyclic chemistry [1]. Pyrrole derivatives have been higher attention in recent times because they could be used in many therapeutic areas such as anti-HIV [2] and antimicrobial agents [3-7].

There have been significant advances in the methodology of multi-component reactions (MCRs) over the past decade and substantial efforts continue to develop new MCRs. The pyrrole ring has found a massive number of therapeutic applications and seems to be found in several natural products [8] Pyrroles tend to build the main framework of porphyrin rings in chlorophyll, heme and vitamin B12, with an increasing medically important drug molecules, like atorvastatin and tolmetin (Fig.1). Several synthetic methods for the construction of pyrroles have been developed till date $[9,10]$.

Among the most common pathways to construction of pyrroles is the Paal-Knorr reaction in which 1,4-dicarbonyl compounds in the presence of primary amines are converted to pyrroles. Some catalysts such as montmorillonite KSF [11], iodine [12], montmorillonite [13], $\mathrm{Zr}\left(\mathrm{KPO}_{4}\right)_{2}$ [14], $\mathrm{Sc}(\mathrm{OTf})_{3}$ [15], alumina [16], microwave irradiation [17,18], ionic liquid [19], $\mathrm{InCl}_{3}$ [20] and $\mathrm{Ga}(\mathrm{OTf})_{3}$ [21] $\mathrm{ZrCl}_{4} / \mathrm{ultrasonic}$ irradiation [22], have been used for this conversion.
Aryl glyoxal containing functional groups such as aldehyde and ketone undergo condensation reaction, play a vital role in organic synthesis, particularly in heterocyclic molecule synthesis. The cost effective and easy method for the synthesis of pyrrole derivatives under mild conditions from a one-pot reaction of aryl glyoxal derivatives and equimolar mixture of 1,3-dicarbonyl compounds in the presence $\mathrm{UO}_{2}\left(\mathrm{NO}_{3}\right)_{2} * 6 \mathrm{H}_{2} \mathrm{O}$ catalyst.
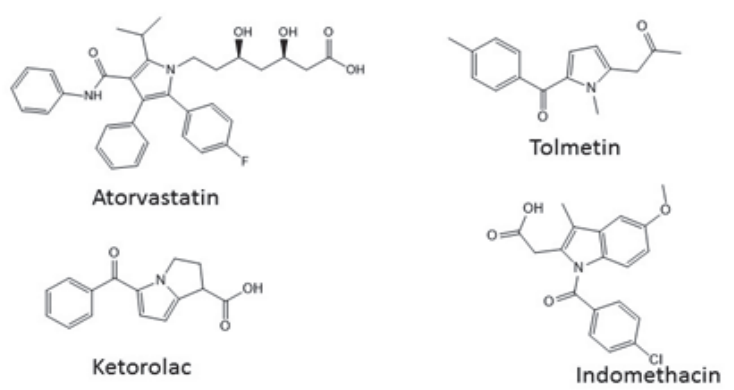

Figure.1 Pharmaceutically active molecules containing pyrrole ring

\section{RESULTS AND DISCUSSION}

As a basic model system, the reaction between aryl glyoxal (1 mmol) with an equimolar amount of 1,3dicarbonyl compound and catalytic amount of uranyl nitrate was investigated to assess the efficiency of the method and enhance the reaction conditions. Choosing a suitable reaction medium is well known to be of crucial importance for efficient synthesis. The mixture of the reactions was tested under various conditions. Solvent and temperature outcomes for this reaction have been measured, and the findings are summarized in Table $1 . \mathrm{UO}_{2}\left(\mathrm{NO}_{3}\right)_{2} \cdot 6 \mathrm{H}_{2} \mathrm{O}$ is a more economical catalyst compared to the other Lewis acid catalysts mentioned in the literature for the synthesis of functionalized pyrrole compounds.

The reaction conditions were efficiently established using condensation of aryl glyoxal and 1,3-dicarbonyl compound 
with catalytic quantities of uranyl nitrate in classical and microwave irradiations using diverse solvent systems such as dichloromethane, chloroform, ethanol, acetonitrile, methanol and various percentage of catalytic mole ratio also examined, and the results were also summarized.<smiles>[R]C(=O)CC([R])=O</smiles>

SCHEME 1 Facile one pot synthesis of functionalized pyrrole derivatives.

Table 1 showing that polar solvents such as methanol, ethanol, and acetonitrile yielded better than nonpolar solvents like chloroform, dichloromethane and the results suggesting that ethanol was the best solvent for this type of conversion. The results show that the reaction under microwave irradiation continues more effectively compared with traditional heating. Additionally, the catalyst loading effect was observed. The maximum percentage of the mole catalyst was $10 \mathrm{~mole} \%$. There was no change in the yield percentage when we increased the catalyst mole percentage.

TABLE I.

EFFECT OF SOLVENT

\begin{tabular}{|c|c|c|c|c|}
\hline S. No & Solvents & $\begin{array}{c}\text { Catalyst } \\
(\mathrm{mol} \%)\end{array}$ & $\begin{array}{c}\text { Time } \\
(\mathrm{min} .)\end{array}$ & $\begin{array}{c}\text { Yield } \\
(\%)\end{array}$ \\
\hline 1 & $\mathrm{CHCl}_{3}$ & 10 & 20 & 16 \\
2 & $\mathrm{DCM}$ & 10 & 20 & 23 \\
3 & Ethanol & 10 & 15 & 85 \\
4 & Methanol & 10 & 18 & 73 \\
5 & Acetonitrile & 10 & 19 & 65 \\
\hline
\end{tabular}

After examination of the optimized reaction conditions, this approach was used for the preparation of pyrrole using different aryl glyoxal and 1,3-dicarbonyl compounds under microwave and traditional heating conditions for the development of new pyrrole compounds in presence of uranyl nitrate catalyst. (Table 3 ). The highly water-soluble catalyst could be isolated by washing with more amount of ice-cold water by filtration. All the constructed derivatives distinguished by IR, elemental analysis, ${ }^{1} \mathrm{H}$ and ${ }^{13} \mathrm{C}$ NMR.

TABLE II.

EFFECT OF CATALYSTS LOADING

\begin{tabular}{|c|c|c|c|c|}
\hline S. No & Catalyst & $\begin{array}{c}\text { Catalyst } \\
(\mathrm{mol} \%)\end{array}$ & $\begin{array}{c}\text { Time } \\
(\mathrm{min} .)\end{array}$ & $\begin{array}{c}\text { Yield } \\
(\%)\end{array}$ \\
\hline 1 & $\mathrm{UO}_{2}\left(\mathrm{NO}_{3}\right)_{2} \cdot 6 \mathrm{H}_{2} \mathrm{O}$ & 5 & 15 & 75 \\
2 & $\mathrm{UO}_{2}\left(\mathrm{NO}_{3}\right)_{2} \cdot 6 \mathrm{H}_{2} \mathrm{O}$ & 10 & 15 & 85 \\
3 & $\mathrm{UO}_{2}\left(\mathrm{NO}_{3}\right)_{2} \cdot 6 \mathrm{H}_{2} \mathrm{O}$ & 15 & 15 & 85 \\
4 & $\mathrm{UO}_{2}\left(\mathrm{NO}_{3}\right)_{2} \cdot 6 \mathrm{H}_{2} \mathrm{O}$ & 20 & 15 & 85 \\
5 & $\mathrm{Conc.} \mathrm{H}_{2} \mathrm{SO}_{4}$ & $1 \mathrm{~mL}$ & $12 \mathrm{~h}$ & 55 \\
\hline
\end{tabular}

\section{EXPERIMENTAL PRocedure}

\section{General protocol for the preparation of} pyrrole derivatives (3a-i):

\section{General Method}

A mixture of aryl glyoxal (1 mmol), 1,3-dicaronyl compound $(1 \mathrm{mmol})$, ammonium acetate $(2 \mathrm{mmol}), 10 \mathrm{~mL}$ of ethanol and uranyl nitrate $(10 \mathrm{~mol}$ percent) was refluxed as shown in Table 3. After TLC indicated completion of the reaction, the reaction blend was transferred into ice-cold water. Stir the mixture for about 15-20 minutes and left 10 hours. The obtained solids were collected by the filtration through a funnel, washed with cold water and then purified from hot ethanol to provide 3a-i pure derivatives.

TABLE III.

EXPERIMENTAL RESULTS AND PHYSICAL DATA OF PYRROLE DERIVATIVES

\begin{tabular}{|c|c|c|c|c|c|c|}
\hline $\begin{array}{c}\text { Comp } \\
\text { ound }\end{array}$ & $\mathrm{R}$ & $\mathrm{R}_{1}$ & $\mathrm{R}_{2}$ & $\begin{array}{c}\text { Reaction } \\
\text { Time } \\
(\mathrm{min})\end{array}$ & $\begin{array}{c}\text { Yield } \\
(\%)\end{array}$ & m.p. $\left({ }^{\circ} \mathrm{C}\right)$ \\
\hline $3 \mathrm{a}$ & $\mathrm{H}$ & $\mathrm{Me}$ & $\mathrm{Me}$ & 15 & 91 & dec. 234 \\
\hline $3 \mathrm{~b}$ & $\mathrm{H}$ & $\mathrm{Me}$ & $\mathrm{OMe}$ & 16 & 90 & dec. 231 \\
\hline $3 \mathrm{c}$ & $\mathrm{H}$ & $\mathrm{Me}$ & $\mathrm{OEt}$ & 17 & 86 & dec. 233 \\
\hline $3 \mathrm{~d}$ & $4-\mathrm{F}$ & $\mathrm{Me}$ & $\mathrm{Me}$ & 18 & 83 & dec. 229 \\
\hline $3 \mathrm{e}$ & $4-\mathrm{F}$ & $\mathrm{Me}$ & $\mathrm{OMe}$ & 18 & 85 & dec. 247 \\
\hline $3 \mathrm{f}$ & $4-\mathrm{F}$ & $\mathrm{Me}$ & $\mathrm{OEt}$ & 19 & 83 & dec. 236 \\
\hline $3 \mathrm{~g}$ & $4-\mathrm{NO}_{2}$ & $\mathrm{Me}$ & $\mathrm{Me}$ & 19 & 83 & dec. 246 \\
\hline $3 \mathrm{~h}$ & $4-\mathrm{NO}_{2}$ & $\mathrm{Me}$ & $\mathrm{OMe}$ & 18 & 86 & dec. 224 \\
\hline $3 \mathrm{i}$ & $4-\mathrm{NO}_{2}$ & $\mathrm{Me}$ & $\mathrm{OEt}$ & 18 & 82 & dec. 243 \\
\hline
\end{tabular}

\section{Microwave irradiation}

In a small beaker, a mixture of aryl glyoxal (1 mmol), 1,3dicaronyl compound (1 mmol), ammonium acetate (2 mmol), uranyl nitrate (10 mol percent) and ethanol $5 \mathrm{~mL}$ was taken and then microwave irradiation was applied to the reaction mixture at an interval of $2 \mathrm{~min}$ at $180 \mathrm{~W}$ for 11-18 minutes summarized in table 3 . The reaction was monitored by TLC. After the reaction was finished, the reaction blend poured into cold water and the separated adduct was filtered, dried, and then purified from hot methanol to provide pure 3a-i compounds, and their physical data are provided in Table 3 .

Compound (3a): mp: Decomp at $234{ }^{\circ} \mathrm{C}$; FT-IR (KBr): v 3370, 3123, 3023, 1676, 1532, 1454, 1387, 1216, $1083 \mathrm{~cm}^{-1}$; ${ }^{1} \mathrm{H}$ NMR (400 MHz, DMSO, ppm), $\delta_{\mathrm{H}}=10.65(\mathrm{~s}, 1 \mathrm{H}, \mathrm{NH})$, $9.23(\mathrm{~s}, 1 \mathrm{H}, \mathrm{OH}), 7.09(\mathrm{~d}, J=7.3 \mathrm{~Hz}, 2 \mathrm{H}, \mathrm{Ar}-\mathrm{H}), 6.45(\mathrm{t}, J=$ $7.0 \mathrm{~Hz}, 2 \mathrm{H}, \mathrm{Ar}-\mathrm{H}), 6.51(\mathrm{t}, J=7.23 \mathrm{~Hz}, 1 \mathrm{H}, \mathrm{Ar}-\mathrm{H}), 2.12(\mathrm{~s}$, $\left.3 \mathrm{H}, \mathrm{CH}_{3}\right), 1.79\left(\mathrm{~s}, 3 \mathrm{H}, \mathrm{CH}_{3}\right) \cdot{ }^{13} \mathrm{C}$ NMR $(100.128 \mathrm{MHz}$, DMSO, ppm), $\delta_{\mathrm{C}}=196.9,142.3,134.4,130.5,127.5,123.3$, 
$121.3,109.4,109.1,22.9,14.4$; Elemental analysis, calculated for $\mathrm{C}_{13} \mathrm{H}_{13} \mathrm{NO}_{2}: \mathrm{C}, 72.54 ; \mathrm{H}, 6.09 ; \mathrm{N}, 6.51$. Experimental: C, 72.51; H, 6.10; N, 6.53\%.

Compound (3b): mp: Decomp at $231{ }^{\circ} \mathrm{C}$; FT-IR (KBr): v $3430,3079,2976,2872,1698,1643,1523,1423$, 1313,1191, $1012 \mathrm{~cm}^{-1}$; ${ }^{1} \mathrm{H}$ NMR (400 MHz, DMSO, ppm), $\delta_{\mathrm{H}}=10.43(\mathrm{~s}, 1 \mathrm{H}, \mathrm{NH}), 8.98(\mathrm{~s}, 1 \mathrm{H}, \mathrm{OH}), 7.34(\mathrm{~m}, 2 \mathrm{H}, \mathrm{Ar}-$ $\mathrm{H}), 7.28(\mathrm{~m}, 3 \mathrm{H}, \mathrm{Ar}-\mathrm{H}), 2.12\left(\mathrm{~s}, 3 \mathrm{H}, \mathrm{CH}_{3}\right), 1.79\left(\mathrm{~s}, 3 \mathrm{H}, \mathrm{CH}_{3}\right)$; ${ }^{13} \mathrm{C}$ NMR (100.128 MHz, DMSO, ppm), $\delta_{\mathrm{C}}=169.4,142.1$, $132.4,131.4,126.5,125.9,121.8,113.7,109.9,61.2,16.2$; Elemental analysis, calculated for $\mathrm{C}_{13} \mathrm{H}_{13} \mathrm{NO}_{3}$ : C, 67.52; H,5.67; N, 6.06. Experimental: C, 67.87; H, 5.65; N, 6.22\%.

Compound (3c): mp: Decomp at $233{ }^{\circ} \mathrm{C}$; FT-IR (KBr): $v$ 3338, 3067, 2978, 1688, 1667, 1523, 1421, 1312, 1178, 1087, $1023 \mathrm{~cm}^{-1} ;{ }^{1} \mathrm{H}$ NMR (400 MHz, DMSO, ppm), $\delta \mathrm{H}=$ $10.23(\mathrm{~s}, 1 \mathrm{H}, \mathrm{NH}), 8.98(\mathrm{~s}, 1 \mathrm{H}, \mathrm{OH}), 7.56(\mathrm{~d}, J=6.9 \mathrm{~Hz}$, $2 \mathrm{H}, \mathrm{Ar}-\mathrm{H}), 7.12(\mathrm{t}, J=7.23 \mathrm{~Hz}, 2 \mathrm{H}, \mathrm{Ar}-\mathrm{H}), 7.06(\mathrm{t}, J=7.12$ $\mathrm{Hz}, 1 \mathrm{H}, \mathrm{Ar}-\mathrm{H}$ ), 3.87 (q, $\left.J=6.98 \mathrm{~Hz}, 2 \mathrm{H}, \mathrm{CH}_{2}\right), 2.45$ (s, 3H, $\left.\mathrm{CH}_{3}\right), 1.42\left(\mathrm{t}, J=6.95 \mathrm{~Hz}, 3 \mathrm{H}, \mathrm{CH}_{3}\right) .{ }^{13} \mathrm{C} \mathrm{NMR}(100.128$ $\mathrm{MHz}, \mathrm{DMSO}, \mathrm{ppm}), \delta_{\mathrm{C}}=169.4,146.7,132.9,131.2,127.6$, 123.9, 121.7, 110.1, 109.7, 60.1, 18.1, 15.2; Elemental analysis, calculated for $\mathrm{C}_{14} \mathrm{H}_{15} \mathrm{NO}_{3}: \mathrm{C}, 68.56 ; \mathrm{H}, 6.16 ; \mathrm{N}$, 5.71. Experimental: C, 68.56; H, 6.09; N, 5.76\%.

Compound (3d): mp: Decomp at $229^{\circ} \mathrm{C}$; FT-IR (KBr): v $3448,3205,3030,1684,1632,1509,1409,1303,1225$, 1168, 1094, $1076 \mathrm{~cm}^{-1}$; ${ }^{1} \mathrm{H}$ NMR (400 MHz, DMSO, ppm), $\delta_{\mathrm{H}}=10.81(\mathrm{~s}, 1 \mathrm{H}, \mathrm{NH}), 8.69(\mathrm{~s}, 1 \mathrm{H}, \mathrm{OH}), 7.67(\mathrm{dd}, J=8.94$ $\mathrm{Hz}, \& J=5.25 \mathrm{~Hz}, 2 \mathrm{H}, \mathrm{Ar}-\mathrm{H}), 7.05(\mathrm{~m}, 2 \mathrm{H}, \mathrm{Ar}-\mathrm{H}) 2.23(\mathrm{~s}$, $\left.3 \mathrm{H}, \mathrm{CH}_{3}\right), 2.05\left(\mathrm{~s}, 3 \mathrm{H}, \mathrm{CH}_{3}\right) ; 13 \mathrm{C} \mathrm{NMR}(100.128 \mathrm{MHz}$, DMSO, ppm), $\delta_{\mathrm{C}}=194.3,165.4,131.4,129.3,127.8,116.4$, $115.9, \quad 112.8, \quad 111.9,25.8,16.9$; Elemental analysis, calculated for $\mathrm{C}_{13} \mathrm{H}_{12} \mathrm{FNO}_{2}: \mathrm{C}, 66.94 ; \mathrm{H}, 5.19 ; \mathrm{N}, 6.01$. Experimental: C, 66.88; H, 5.14; N, 6.06\%.

Compound (3e): mp: Decomp at $247{ }^{\circ} \mathrm{C}$; FT-IR (KBr): v 3325, 3067, 2976, 1703, 1659, 1514, 1505, 1489, 1314, 1218, 1158, 1098, $1080 \mathrm{~cm}^{-1} ;{ }^{1} \mathrm{H}$ NMR (400 MHz, DMSO, $\mathrm{ppm}), \delta_{\mathrm{H}}=10.43(\mathrm{~s}, 1 \mathrm{H}, \mathrm{NH}), 8.65(\mathrm{~s}, 1 \mathrm{H}, \mathrm{OH}), 7.64(\mathrm{~m}$, $2 \mathrm{H}, \mathrm{Ar}-\mathrm{H}), 7.09$ (t, $J=7.9 \mathrm{~Hz}, 2 \mathrm{H}, \mathrm{Ar}-\mathrm{H}), 3.28\left(\mathrm{~s}, 3 \mathrm{H}, \mathrm{CH}_{3}\right)$, $2.24\left(\mathrm{~s}, 3 \mathrm{H}, \mathrm{CH}_{3}\right) ;{ }^{13} \mathrm{C}$ NMR (100.128 MHz, DMSO, ppm), $\delta_{\mathrm{C}}=168.5,161.9,134.9,131.2,126.8,120.0,115.9,112.8$ $111.9,52.4,18.1$; Elemental analysis, calculated for $\mathrm{C}_{13} \mathrm{H}_{12} \mathrm{FNO}_{3}$ : C, 62.65; H, 4.85; N, 5.62. Experimental: C, 62.76; H, 4.89; N, 5.39\%.

Compound (3f): $\mathrm{mp}$; Decomp at $236{ }^{\circ} \mathrm{C}$; FT-IR (KBr): v $3345,3086,3010,1738,1548,1545,1528,1329,1252$, 1173, 1102, 1084, $1028 \mathrm{~cm}^{-1}$; ${ }^{1} \mathrm{H}$ NMR (400 MHz, DMSO, ppm), $\delta_{\mathrm{H}}=10.32(\mathrm{~s}, 1 \mathrm{H}, \mathrm{NH}), 8.37(\mathrm{~s}, 1 \mathrm{H}, \mathrm{OH}), 7.67(\mathrm{dd}, J$ $=5.4 \mathrm{~Hz}, J=7.6 \mathrm{~Hz} 2 \mathrm{H}, \mathrm{Ar}-\mathrm{H}), 7.08(\mathrm{~m}, 2 \mathrm{H}, \mathrm{Ar}-\mathrm{H}), 3.92$ $\left(\mathrm{q}, J=6.78 \mathrm{~Hz}, 2 \mathrm{H}, \mathrm{CH}_{2}\right), 2.29\left(\mathrm{~s}, 3 \mathrm{H}, \mathrm{CH}_{3}\right), 1.06(\mathrm{t}, J=6.9$ $\left.\mathrm{Hz} 3 \mathrm{H}, \mathrm{CH}_{3}\right) ;{ }^{13} \mathrm{C}$ NMR (100.128 MHz, DMSO, ppm), $\delta_{\mathrm{C}}=$ $167.9,161.9,131.4,131.0,129.2,117.4,115.9,112.8$, $111.7,52.3,18.3,13.9$. Elemental analysis, calculated for $\mathrm{C}_{14} \mathrm{H}_{14} \mathrm{FNO}_{3}$ : C, 63.87; H, 5.36; N, 5.32. Experimental: C, $63.83 ; \mathrm{H}, 5.45 ; \mathrm{N}, 5.32 \%$.
Compound (3g): $\mathrm{mp}$; Decomp at $246{ }^{\circ} \mathrm{C}$; FT-IR (KBr): v 3482, 3345, 1647, 1583, 1511, 1318, 1213, 1176, 1098, $1043,1011 \mathrm{~cm}^{-1}$; ${ }^{1} \mathrm{H}$ NMR (400 MHz, DMSO, ppm), $\delta_{\mathrm{H}}=$ 11.12 (s, 1H, NH) 10.43 (s, $1 \mathrm{H}, \mathrm{OH}), 8.17$ (d, $J=8.8 \mathrm{~Hz}$, 2H, Ar-H), 7.67 (d, $J=8.2 \mathrm{~Hz}, 2 \mathrm{H}, \mathrm{Ar}-\mathrm{H}), 2.42$ (s, 3H, $\left.\mathrm{CH}_{3}\right), 2.12\left(\mathrm{~s}, 3 \mathrm{H}, \mathrm{CH}_{3}\right) ;{ }^{13} \mathrm{C}$ NMR $(100.128 \mathrm{MHz}, \mathrm{DMSO}$, $\mathrm{ppm}), \delta_{\mathrm{C}}=197.3,148.6,142.6,138.9,134.2,123.6,121.5$, 109.8, 196.7, 28.7, 14.9; Elemental analysis, calculated $\mathrm{C}_{13} \mathrm{H}_{12} \mathrm{~N}_{2} \mathrm{O}_{4}$ : C, 60.00; H, 4.65; N, 10.76\%. Experimental: C, $60.23 ; \mathrm{H}, 4.35 ; \mathrm{N}, 10.54 \%$.

Compound (3h): $\mathrm{mp}$; Decomp at $224^{\circ} \mathrm{C}$; FT-IR (KBr): v 3523, 3432, 1676, 1621, 1512, 1312, 1232, 1163, 1198, 1056, $1031 \mathrm{~cm}^{-1}$; ${ }^{1} \mathrm{H}$ NMR (400 MHz, DMSO, ppm), $\delta_{\mathrm{H}}=$ 11.43 (s, 1H, NH), 8.98 (s, 1H, OH), 8.18 (d, $J=8.6 \mathrm{~Hz}$, 2H, Ar-H), 7.87 (d, $J=8.9 \mathrm{~Hz}, 2 \mathrm{H}, \mathrm{Ar}-\mathrm{H}), 3.76(\mathrm{~s}, 3 \mathrm{H}$, $\left.\mathrm{CH}_{3}\right), 2.34\left(\mathrm{~s}, 3 \mathrm{H}, \mathrm{CH}_{3}\right) ;{ }^{13} \mathrm{C}$ NMR $(100.128 \mathrm{MHz}, \mathrm{DMSO}$, ppm), $\delta_{\mathrm{C}}=168.7,148.9,142.3,139.6,135.7,123.7,121.9$, 109.6, 100.7, 50.7, 13.9; Elemental analysis, calculated for $\mathrm{C}_{13} \mathrm{H}_{12} \mathrm{~N}_{2} \mathrm{O}_{5}$ : C, 56.52; H, 4.38; N, 10.14\%. Experimental: C, 56.34; H, 4.45; N, 10.48\%.

Compound (3i): $\mathrm{mp}$; Decomp at $243{ }^{\circ} \mathrm{C}$; FT-IR (KBr): $v$ 3498, 3434, 1683, 1589, 1532, 1323, 1212, 1178, 1102, 1054, $1023 \mathrm{~cm}^{-1}$; ${ }^{1} \mathrm{H}$ NMR (400 MHz, DMSO, ppm), $\delta_{\mathrm{H}}=$ $11.65(\mathrm{~s}, 1 \mathrm{H}, \mathrm{NH}), 9.05(\mathrm{~s}, 1 \mathrm{H}, \mathrm{OH}), 8.19(\mathrm{~d}, J=8.3 \mathrm{~Hz}$, 2H, Ar-H), 7.79 (d, $J=8.7 \mathrm{~Hz}, 2 \mathrm{H}, \operatorname{Ar}-\mathrm{H}), 4.29$ (q, $J=6.9$ $\left.\mathrm{Hz}, 2 \mathrm{H}, \mathrm{CH}_{2}\right), 2.23\left(\mathrm{~s}, 3 \mathrm{H}, \mathrm{CH}_{3}\right), 1.23(\mathrm{t}, J=6.7 \mathrm{~Hz}, 3 \mathrm{H}$, $\left.\mathrm{CH}_{3}\right) ;{ }^{13} \mathrm{C}$ NMR $(100.128 \mathrm{MHz}, \mathrm{DMSO}, \mathrm{ppm}), \delta_{\mathrm{C}}=168.5$, 148.6, 144.3, 139.7, 135.7, 123.8, 121.9, 111.5, 100.3, 61.2, 14.9, 14.0; Elemental analysis, calculated for $\mathrm{C}_{14} \mathrm{H}_{14} \mathrm{~N}_{2} \mathrm{O}_{5}$ : C, 57.93; H, 4.86; N, 9.65\%. Experimental: C, 57.99; H, $4.79 ; \mathrm{N}, 9.89 \%$.

\section{CONCLUSIONS}

A simple and effective method for the synthesis of acetyl4-hydroxy-2-methyl-5-phenyl-1H-pyrrole derivatives was proposed using $\mathrm{UO}_{2}\left(\mathrm{NO}_{3}\right)_{2} \cdot 6 \mathrm{H}_{2} \mathrm{O}$ as a catalyst for the condensation of various substituted aryl glyoxal, 1,3dicarbonyl compounds and ammonium acetate under traditional heating and microwave irradiation conditions. The profits of this technique are operational simplicity, short reaction time, easy work-up, easily available catalyst and, high conversion of yields.

\section{REFERENCES}

[1] E. Y. Schmidt, A. I. Mikhaleva, A. M. Vasiltsov, A. B Zaitsev and N. V. Zorina, Arkivoc., 2005, 7, 11.

[2] S. Jiang, H. Lu, S. Liu, Q. Zhao, Y. He and A. K. Debnath, Antimicrob. Agents Chemother., 2004, 48, 4349.

[3] W. Burli, D. McMinn, J. A. Kaizerman, W. Hu, Y. Ge, Q. Pack, V. Jiang, M. Gross, M. Garcia, R. Tanaka and H. E. Moser, Bioorg. Med. Chem. Lett., 2004, 14, 1253.

[4] M. S. Mohamed, R. Kamel and S. S. Fatahala, Eur. J. Med. Chem., 2010, 45, 2994.

[5] R. R. Khanwelkar, G. S. Chen, C. N. Chang, H. C. Hsu, H. C. Lin, Y. C. Shih, S. H. Chou, H. W. Tseng, C. P. Liu, C. M. Tu, T. L. Hu, Y. J. Tsai and J. W. Chern, Bioorg. Med. Chem. Lett., 2010, 18, 4674 
[6] J. Lehuede, B. Fauconneau, L. Barrier, M. Ourakow, A. Piriou and J. M. Vierfond, Eur. J. Med. Chem., 1999, 34, 991.

[7] A. Tafi, R. Costi, M. Botta, R. D. Santo, F. Corelli, S. Massa, A. Ciacci, F. Manetti and M. Artico, J. Med. Chem., 2002, 45, 2720.

[8] A. Trost, A. Gutierrez and R. Livingston, Org. Lett., 2009, $11,2539$.

[9] L. Wen, Y. Shi and G. Liu, Org. Chem., 2012, 77, 4252.

[10] D. Hong, Y. Zhu, Y. Li, X. Lin, P. Lu and Y. Wang, Org. Lett., 2011, 13, 4668.

[11] R. Ballini, L. Barboni, G. Bosica and M. Petrini, Synlett., $2000,3,391$.

[12] S. Samadjar, F. F. Becker and B. K. Banik, Heterocycles., $2001,55,1019$.

[13] B.K. Banik, S. Samajdar, I. Banik, J. Org. Chem., 2004, 69, 213.
[14] G. Song, B. Wang, G. Wang, Y. Kang, T. Yang and L. Yang, Synth. Commun., 2005, 35, 1051.

[15] M. Curini, F. Montanari, O. Rosati, E. Lioy and R. Margarita, Tetrahedron Lett., 2003, 44, 3923.

[16] B. Wang, Y. Gu, C. Luo, T. Yang, L. Yang and J. Suo, Tetrahedron Lett., 2004, 45, 3417.

[17] J. Chen, H. Wu, Z. Zheng, C. Jin, X. Zhang and W. Su, Tetrahedron Lett., 2006, 47, 5383.

[18] T. N. Danks, Tetrahedron Lett., 1999, 40, 3957.

[19] K. Venkatesan, CVR Journal of Science and Technology., $2018,14,112$.

[20] Z. H. Zhang, J. J. Li and T. S. Li, Ultrason. Sonochem., $2008,15,673$.

[21] J. X. Chen, M. C. Liu, X. L. Yang, J. C. Ding and H. Y. Wu, J. Braz. Chem. Soc., 2008, 19, 877.

[22] J. Chen, X. Yang, M. Liu, H. Wu, J. Ding and W. Su, Synth. Commun., 2009, 39, 4180. 